\title{
The Chemistry Preprint Server: An Experiment in Scientific Communication
}

James Weeks, ChemWeb Inc.

84 Theobalds Road, Holborn, London WC1X 8RR

James.Weeks@ChemWeb.com

WORKSHOP ON OAI AND PEER REVIEW JOURNALS IN EUROPE

Geneva, 22 ${ }^{\text {nd }}$ March 2001 


\section{Outline of Presentation}

- Introduction to ChemWeb.com

$\checkmark$ Chemistry Preprint Server

- Overview

- Submission procedure

- Browsing and searching

- Where we are now

- Future Directions

- Conclusions

- Questions \& Discussion 


\section{ChemWeb.com}

- Online resource for the chemical community

- Free registration and membership

- Access to essential services and information

- Journals, Databases, The Alchemist Magazine, Shopping Mall, Conference Centre, Careers Centre, Forums

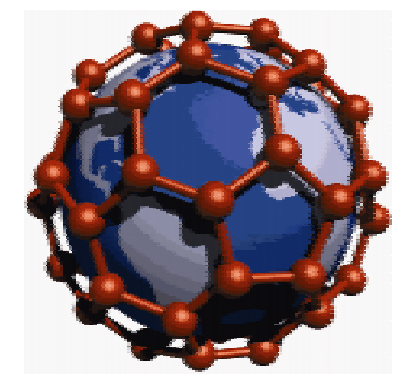




\section{ChemWeb.com}

- Offer free services - temporary \& permanent

- Access to journals

- AutoNom and Beilstein Abstracts

- Encourage member interaction - e.g. forums

- Exploit web technology and functionality - e.g. structure searching

- Over 250,000 members, 2.5 million page impressions per month

Ideal host for the Chemistry Preprint Server! 


\section{Chemistry Preprint Server}

- Freely available and permanent web archive and distribution medium for research articles

- Scientific articles freely available for public access prior to formal review

- Preprint NOT prepublication

- The first Chemistry Preprint Server!

- Modelled on very successful arXiv preprint server, run by LANL, for mathematics and physics

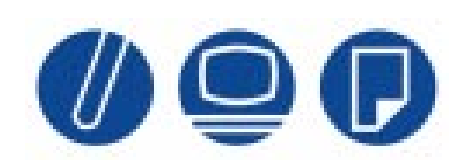

http://preprint.chemweb.com 


\section{Launch of the CPS}

- Launched 21 August 2000 at the ACS National Meeting

- 20 Preprints submitted prior to launch

- Positive response at the meeting

- Primary concerns were issues of prior publication and quality control 


\section{CPS Procedure (1)}

- Author uploads preprint:

- Article, meta-data (abstract, author details etc) \& supplementary files submitted

- File types supported include PDF, MS Word, Postscript, RTF and HTML

- Author selects classification

- Automatic conversion to PDF

- Article screened by 'editor'

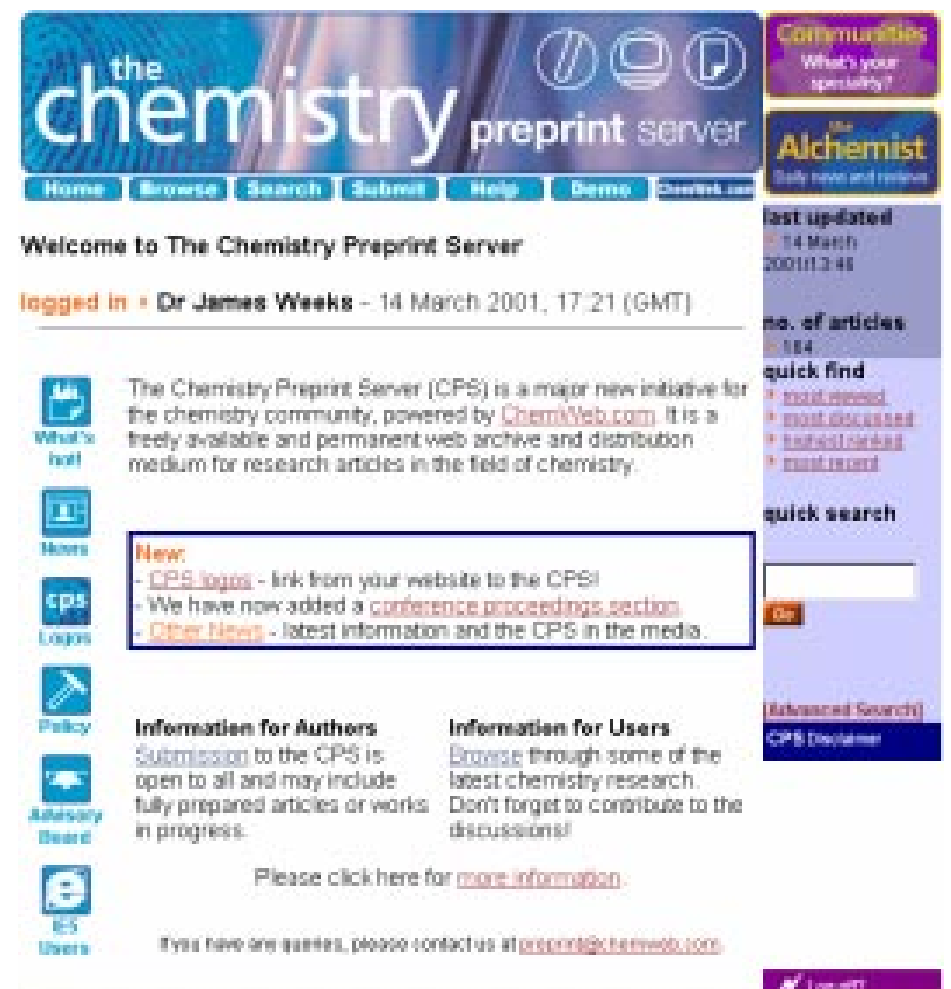

- Author revisions possible (version control)

- Option to redirect to online version at publisher's site following publication 


\section{CPS Procedure (2)}

10 Classifications:

- Analytical Chemistry

- Biochemistry

- Chemical Engineering

- Environmental Chemistry

- Inorganic Chemistry

- Macromolecular Chemistry

- Medicinal Chemistry

- Organic Chemistry

- Physical Chemistry

- Miscellaneous

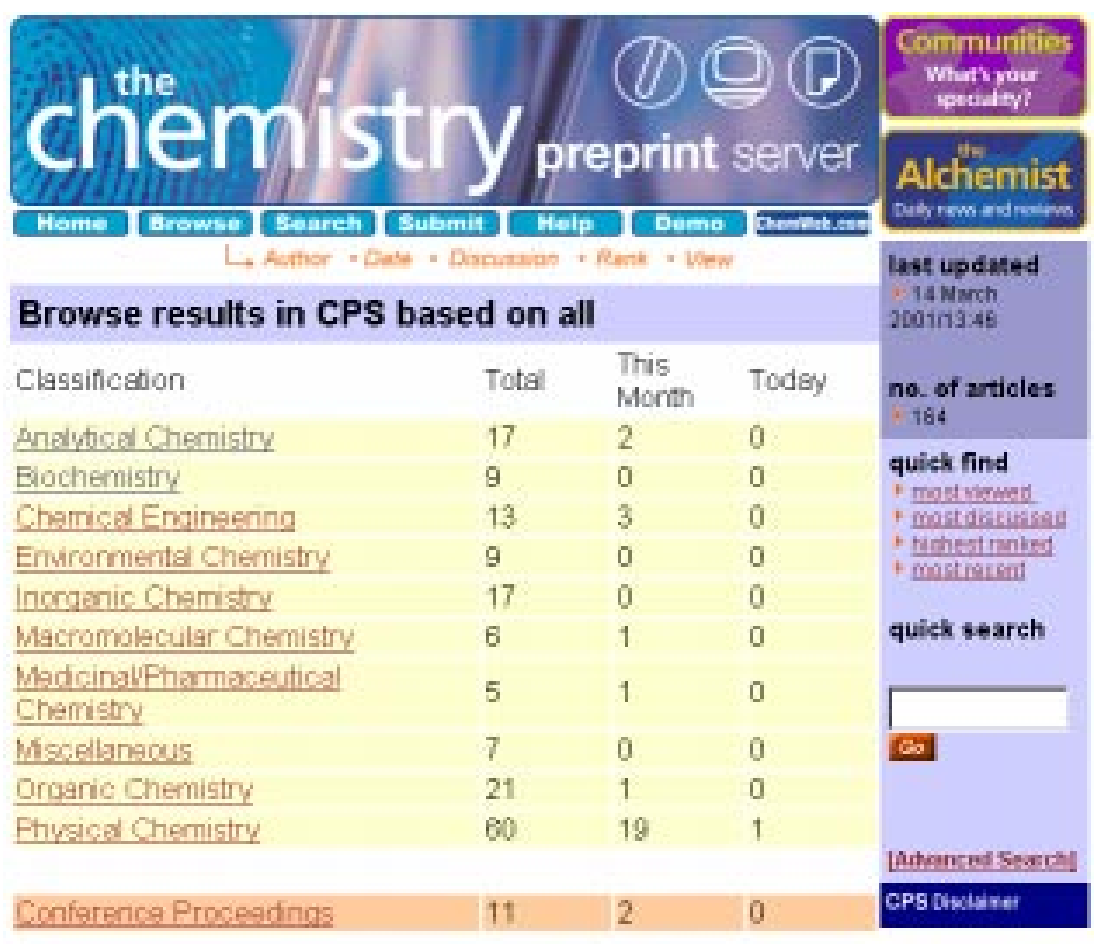

Show mrv email slerts

- New Conference Proceedings section

- Submit pre-conference abstracts or post-conference reports 


\section{CPS Procedure (3)}

- Every article is given a unique identifier

- "Friendly URL" \& Citation Reference

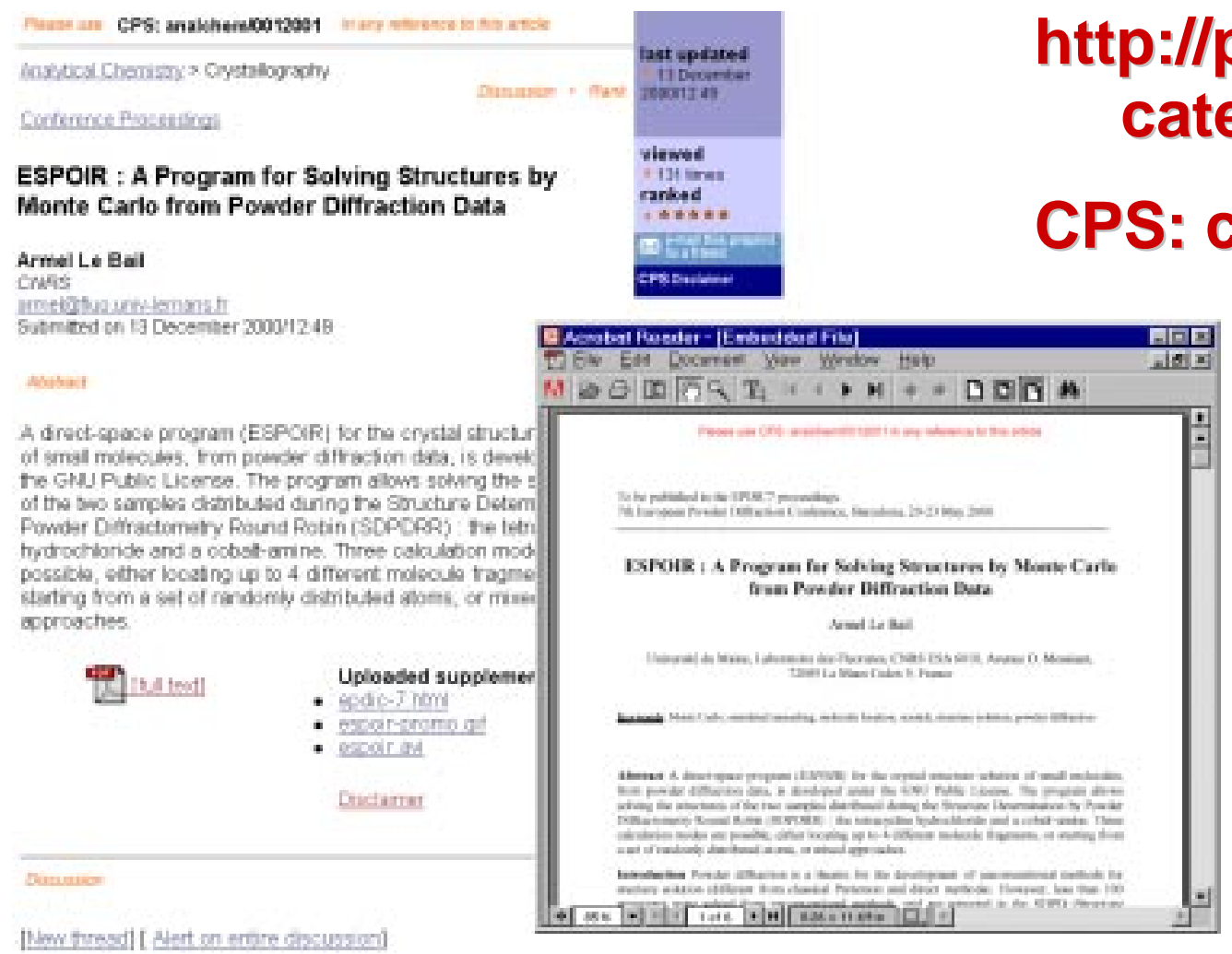




\section{CPS Procedure (4)}

- Users can discuss \& rank articles

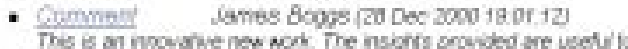
both expermentslits and theoretiong

Bask fakt ancomment

Please select tom to drop dowe menu the number of stars you

trink this preprint deserves

Giverank: a

$\checkmark$ Quick and advanced searching and browsing

- Comprehensive searching across all fields

- Users can access most recent, most viewed, most discussed \&

Search Preprints

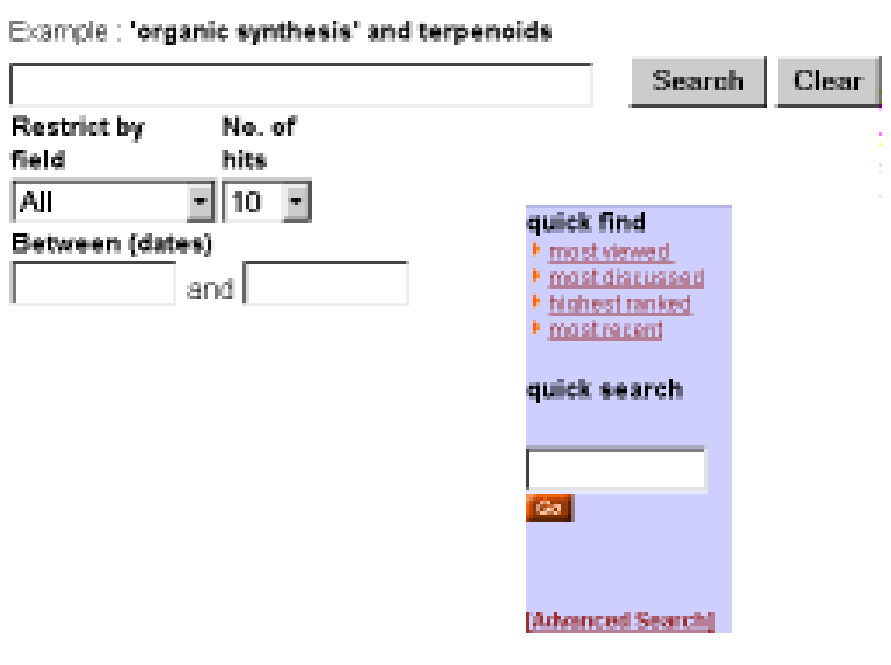
highest ranked articles 


\section{CPS Procedure (5)}

- Individual user profile - email alerts:

- Searches for key words and phrases

- New articles added to a particular classification

- New threads added into a discussion group

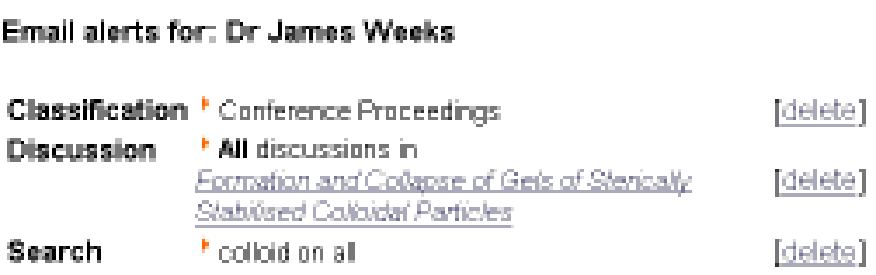

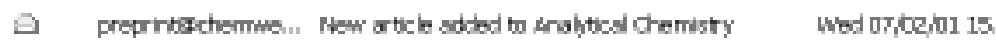

- Recommend preprint to Authors: Armel Le Bai a friend 


\section{CPS - Where We Are Now (1)}

- Over 160 articles submitted in all ten categories

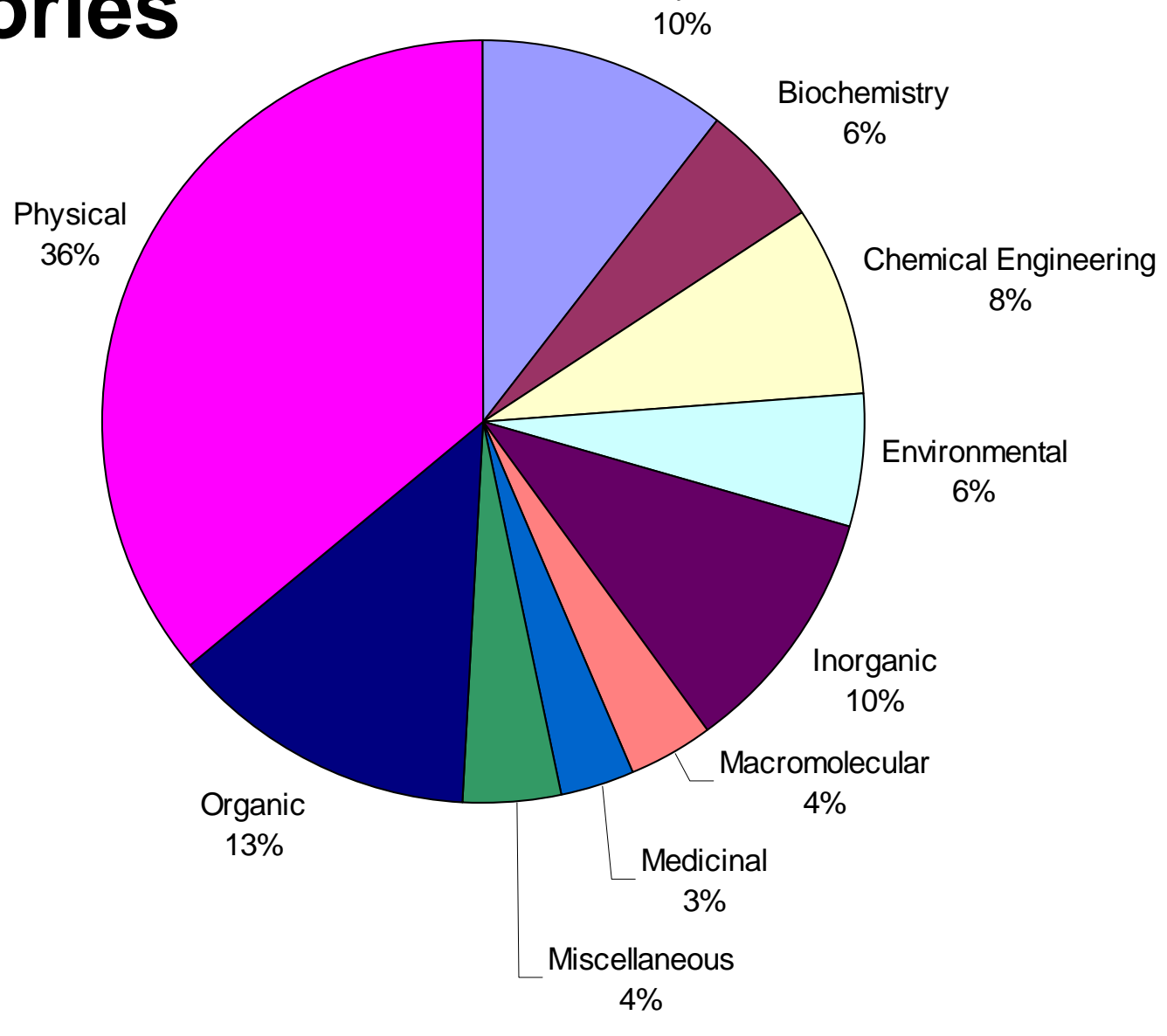

Total number of preprints $=163$

$\square$ Analytical $\square$ Biochemistry $\square$ Chemical Engineering $\square$ Environmental - Inorganic $\square$ Macromolecular $\square$ Medicinal

$\square$ Miscellaneous

- Organic

$\square$ Physical 


\section{CPS - Where We Are Now (2)}

\section{- World-wide appeal to the chemistry community}

Bulgaria

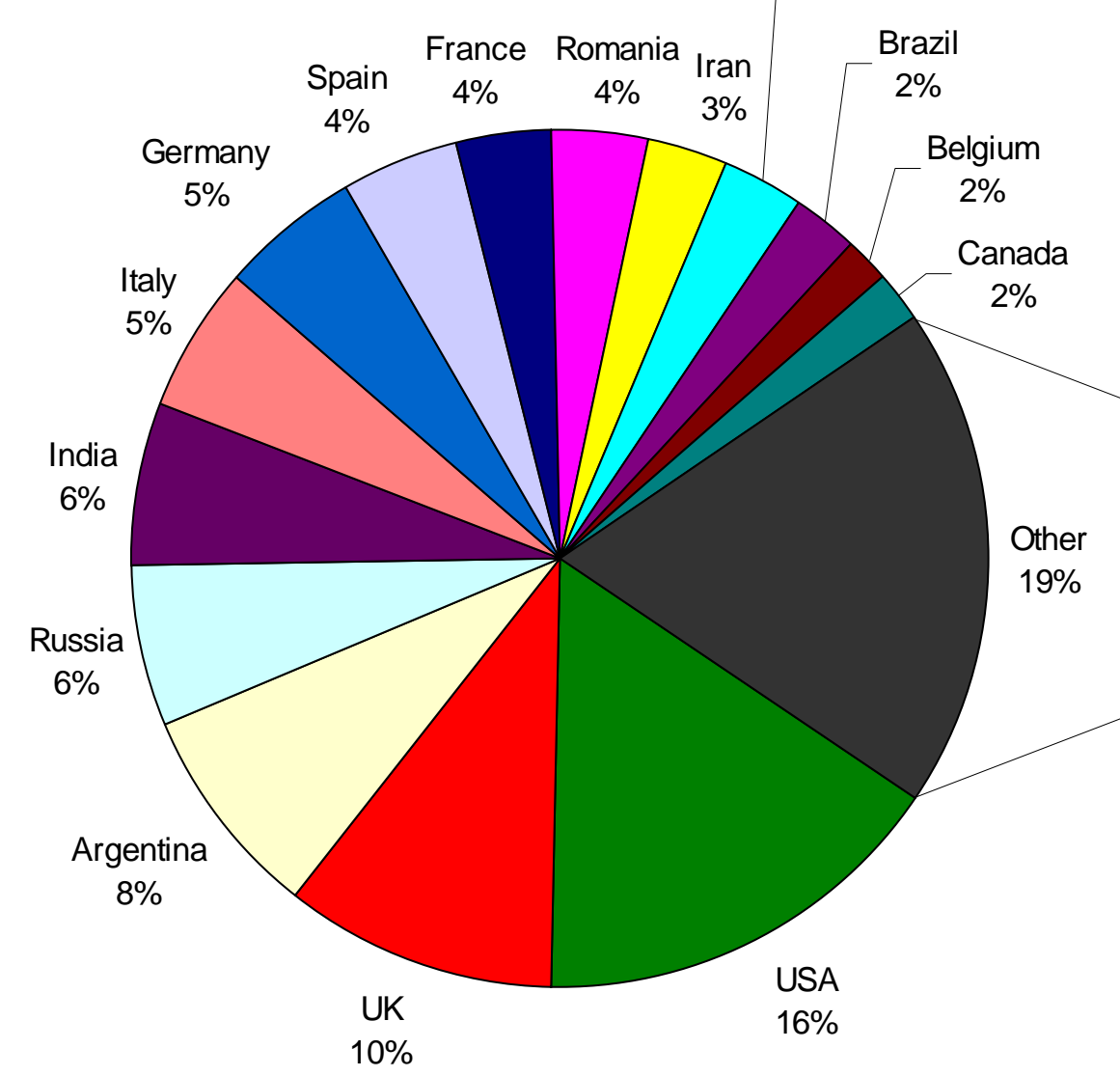

\begin{tabular}{|c|c|}
\hline $\begin{array}{l}\square \text { USA } \\
\square \text { Argentina } \\
\square \text { India } \\
\square \text { Germany } \\
\square \text { France } \\
\square \text { Iran } \\
\square \text { Brazil } \\
\square \text { Canada } \\
\square \text { Sweden } \\
\square \text { Israel } \\
\square \text { Poland } \\
\square \text { Lithuania } \\
\square \text { Egypt } \\
\square \text { Marocco } \\
\square \text { Japan } \\
\square \text { Eire } \\
\square \text { Switzerland } \\
\square \text { Croatia } \\
\square \text { Norway } \\
\square \text { Tunisia }\end{array}$ & $\begin{array}{l}\square \text { UK } \\
\square \text { Russia } \\
\square \text { Italy } \\
\square \text { Spain } \\
\square \text { Romania } \\
\square \text { Bulgaria } \\
\square \text { Belgium } \\
\square \text { Malaysia } \\
\square \text { China } \\
\square \text { South Africa } \\
\square \text { New Zealand } \\
\square \text { Hungary } \\
\square \text { Cuba } \\
\square \text { Finland } \\
\square \text { Netherlands } \\
\square \text { Czech Republic } \\
\square \text { Armenia } \\
\square \text { Ukraine } \\
\square \text { Australia }\end{array}$ \\
\hline
\end{tabular}




\section{CPS - Where We Are Now (3)}

- Each of the top 20 "most viewed" preprints has been viewed by more than 500 individuals

- Top article has over 2000 viewings

- Over 100,000 page impressions per month

- Limited postings to the online discussions 


\section{Future Directions}

\section{Compliance with Santa Fe Convention for Open Archives Initiative}

- Implement meta-data protocols in the immediate future

- Harvest data from Los Alamos (arXiv) preprint server

- US Department of Energy initiative to develop search engine for preprints (http://www.osti.gov/preprints/index.html)

- Automatic submission of preprints to journals

- Author selects journal (publisher-neutral) for article submission when satisfied the article is complete

- Access the CPS on WAP and Palm devices 


\section{Conclusions}

- At present, the CPS is still an experiment in scientific communication

- Early signs indicate a very positive response from the chemistry community

- Further enhancement: compliance with the Open Archives Initiative

- Exciting possibilities for future development, further enhancing chemistry communication 


\title{
Thank you for your attention!
}

\author{
James.Weeks@ChemWeb.com \\ http://preprint.chemweb.com
}

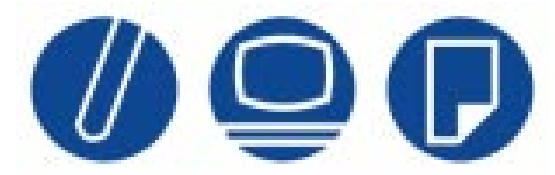

http://preprint.chemweb.com

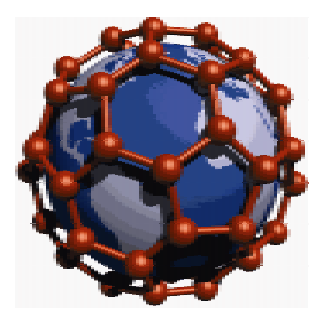

ChemWeb.com 